BUHEP-98-10

hep-ph/9806287

\title{
A Heavy Top Quark From Flavor-Universal Colorons
}

\author{
Marko B. Popovic and Elizabeth H. Simmons * \\ Department of Physics, Boston University, \\ 590 Commonwealth Ave., Boston MA 02215
}

April 16, 2018

\begin{abstract}
Ordinary technicolor and extended technicolor cannot produce the heavy top quark unaided. We demonstrate that a flavor-universal extension of the color interactions combined with an extended hypercharge sector that singles out the third generation can provide the necessary assistance. We discuss current experimental constraints and suggest how collider experiments can search for the predicted new heavy gauge bosons.
\end{abstract}

*e-mail addresses: markopop@buphy.bu.edu, simmons@bu.edu. 


\section{Introduction}

Generating mass through strong gauge dynamics is a challenge. While a technicolor [1] gauge sector can provide appropriate masses for the electroweak gauge bosons by breaking the chiral symmetries of technicolored fermions, explaining the masses and mixings of the quarks and leptons has proven more difficult. Extended technicolor (ETC) models [2] postulate an enlarged gauge group coupling the quarks and leptons to the technifermion condensate, enabling them to acquire mass. The simplest models of this type tend to produce large flavor-changing neutral currents [2] and, if the heavy top quark mass is generated by ETC interactions, excessive weak isospin violation [3] and contributions to $R_{b}$ 沟. Substantially raising the scale at which extended technicolor breaks to its technicolor subgroup can alleviate some of these problems - but renders the model incapable of naturally producing quark masses larger than a few $\mathrm{GeV}$.

Given the large value of the top quark's mass $\left(m_{t} \approx 175 \mathrm{GeV}[5]\right)$ and the sizable splitting between the masses of the top and bottom quarks, it is natural to wonder whether $m_{t}$ has a different origin than the masses of the other quarks and leptons. A variety of dynamical models that exploit this idea have been proposed. Key examples are the dynamical models of 'top-mode' mass generation in which top quark self-interactions drive all of electroweak symmetry breaking [6]. Related to those are the topcolor [7] and topcolor-assisted technicolor [8] models [9, 10] in which the top quark feels different color and hypercharge interactions than other quarks; as a consequence, a top quark condensate enhances the top quark's mass. Finally, there are the non-commuting ETC scenarios where the top quark has weak and extended technicolor interactions different from those of other quarks [11]. The conclusion of these investigations has been that new dynamics peculiar to the top quark can certainly create a large top quark mass. It may even possible to do so while creating a model that accords reasonably well with electroweak precision data.

In this paper, we discuss a variant class of models of dynamical top quark mass generation in which the large mass comes from top-specific gauge interactions. What sets these theories apart is that the top quark differs from the other quarks only in its hypercharge interactions. The extended color interactions are flavor-universal, just as in the coloron model of [12]; the weak interactions display ordinary Cabibbo universality.

After introducing the class of models in section 2 and showing, in section 3 , that the low-energy dynamics admit the possibility of top quark condensation and a large top quark mass, we focus on experimental constraints. Section 4 discusses the phenomenology of the low-energy effective theory, while section 5 explores the possibility of direct searches for the additional massive gauge bosons in the theory.

We note that the physics discussed here must be part of some larger (e.g. ETC) structure at high energies which will create the masses and mixings of the light fermions and produce condensates that break our extended gauge symmetries to 
their standard model subgroups. However, we focus on exploring the dominant effects of the new physics that produces the top quark mass. A discussion of higherscale operators that break all fermion chiral symmetries, account for generational mixing and produce relevant symmetry-breaking condensates may be found in [13].

\section{The Class of Models}

Our models have a gauge structure like that of the original topcolor-assisted technicolor models [8]. Far above the electroweak scale, the gauge group is

$$
S U(N)_{T C} \times S U(3)_{1} \times S U(3)_{2} \times S U(2)_{W} \times U(1)_{1} \times U(1)_{2}
$$

with coupling constants $g_{N}, g_{3_{(1)}}, g_{3_{(2)}}, g_{2}, g_{1_{(1)}}$, and $g_{1_{(2)}}$ respectively. We take the first $S U(3)$ and $U(1)$ groups to have the stronger couplings: $g_{3_{(1)}}>g_{3_{(2)}}$ and $g_{1_{(1)}}>g_{1_{(2)}}$. The group $S U(N)_{T C}$ is the technicolor gauge group.

At an energy scale $\Lambda$, a condensate $\langle\phi\rangle$ transforming under the initial symmetry group as $(1,3, \overline{3}, 1, p,-p)$ breaks the color sector $\left(S U(3)_{1} \times S U(3)_{2}\right)$ to its diagonal subgroup $\left(S U(3)_{C}\right)$ and similarly breaks the hypercharge groups in the pattern $\left(U(1)_{1} \times U(1)_{2} \rightarrow U(1)_{Y}\right)$. The gauge symmetry is reduced to that of the standard model plus the unbroken technicolor group:

$$
S U(N)_{T C} \times S U(3)_{C} \times S U(2)_{W} \times U(1)_{Y} .
$$

At the weak scale, $\Lambda_{E W}<\Lambda$, the technicolor force becomes strong enough to break the chiral symmetries of a set of technifermions and cause electroweak symmetry breaking $S U(2)_{W} \times U(1)_{Y} \rightarrow U(1)_{E M}$. Thus the low-energy gauge boson spectrum includes the massless photon and gluons, the massive $W$ 's and $Z$, and two additional kinds of massive states: an octet of colorons and a single $Z^{\prime}$.

At low energies, the mass eigenstate fields in the color sector (colorons $C^{a}$ and gluons $G^{a}$ ) are related to the original $S U(3)_{1} \times S U(3)_{2}$ gauge fields (denoted $X_{(n)}$ ) via [14]:

$$
G^{a}=\frac{g_{3_{(2)}} X_{(1)}^{a}+g_{3_{(1)}} X_{(2)}^{a}}{\sqrt{g_{3_{(1)}}^{2}+g_{3_{(2)}}^{2}}} \quad \quad C^{a}=\frac{g_{3_{(1)}} X_{(1)}^{a}-g_{3_{(2)}} X_{(2)}^{a}}{\sqrt{g_{3_{(1)}}^{2}+g_{3_{(2)}}^{2}}}
$$

Similar relations hold in the hypercharge sector. The familiar QCD and hypercharge gauge coupling constants are related to the high energy couplings by

$$
\frac{1}{g_{3}^{2}}=\frac{1}{g_{3_{(1)}}^{2}}+\frac{1}{g_{3_{(2)}}^{2}} \quad \frac{1}{g_{1}^{2}}=\frac{1}{g_{1_{(1)}}^{2}}+\frac{1}{g_{1_{(2)}}^{2}}
$$

and their respective fine-structure constants are $\alpha_{Y} \equiv g_{1}^{2} / 4 \pi$ and $\alpha_{s} \equiv g_{3}^{2} / 4 \pi$. The tree level masses of the colorons and $Z^{\prime}$ are

$$
M_{C}=\langle\phi\rangle \sqrt{g_{3_{(1)}}^{2}+g_{3_{(2)}}^{2}} \quad M_{Z^{\prime}}=\langle\phi\rangle|p| \sqrt{g_{1_{(1)}^{2}+g_{1_{(2)}}^{2}}^{2}} .
$$


Note the dependence of the $Z^{\prime}$ mass on the $U(1)$ charges of the condensate $\langle\phi\rangle$.

The gauge transformation properties of the quarks and leptons, which are summarized in Table 1, are significantly different from those in topcolor-assisted technicolor [8]. In the color sector, all quarks transform only under the stronger $S U(3)_{1}$ group, as in the flavor-universal coloron model [12]. In the hypercharge sector only the third family of fermions transforms under the stronger $U(1)_{1}$ and the first two families transform under the weaker $U(1)_{2}$ (all of them with standard model hypercharge assignments). All of the quarks and leptons have the same weak charge assignments as in the standard model. Each generation of ordinary fermions forms an anomaly-free representation of the gauge group (2.1).

As we shall explore in more detail, this set of gauge charge assignments for the fermions still allows natural dynamical generation of a large mass for the top quark (and only the top quark). Yet it leads to a phenomenology differing from that of topcolor-assisted technicolor [8, 10].

\begin{tabular}{|c||c|c|c|c|c|c||}
\hline \hline & $S U(N)_{T C}$ & $S U(3)_{1}$ & $S U(3)_{2}$ & $S U(2)$ & $U(1)_{1}$ & $U(1)_{2}$ \\
\hline \hline I & 1 & $\mathrm{SM}$ & 1 & $\mathrm{SM}$ & 0 & $\mathrm{SM}$ \\
II & 1 & $\mathrm{SM}$ & 1 & $\mathrm{SM}$ & 0 & $\mathrm{SM}$ \\
$\mathrm{III}$ & 1 & $\mathrm{SM}$ & 1 & $\mathrm{SM}$ & $\mathrm{SM}$ & 0 \\
\hline \hline
\end{tabular}

Table 1: Quark and lepton gauge charge assignments for generations I, II and III. An entry of 'SM' indicates that the particles carry the same charges under the given group as they would under the standard model group of the same rank.

\section{Low energy effective theory}

Below the symmetry-breaking scale, $\Lambda$, for the extended color and hypercharge sectors, the interactions among quarks and leptons that arise from exchange of the massive colorons and $Z^{\prime}$ are well-approximated by effective four-fermion interactions

$$
\begin{aligned}
\mathcal{L}_{C} & =-\frac{2 \pi \kappa_{3}}{M_{C}^{2}}\left(\bar{q} \gamma^{\mu} \frac{\lambda^{a}}{2} q\right)\left(\bar{q} \gamma_{\mu} \frac{\lambda_{a}}{2} q\right) \\
\mathcal{L}_{Z^{\prime}}= & -\frac{2 \pi}{M_{Z^{\prime}}^{2}} \frac{\alpha_{Y}^{2}}{\kappa_{1}}\left(\bar{f}_{I I} \gamma^{\mu} \frac{Y}{2} f_{I I}\right)\left(\bar{f}_{I I} \gamma_{\mu} \frac{Y}{2} f_{I I}\right) \\
& -\frac{2 \pi \kappa_{1}}{M_{Z^{\prime}}^{2}}\left(\bar{f}_{I I I} \gamma^{\mu} \frac{Y}{2} f_{I I I}\right)\left(\bar{f}_{I I I} \gamma_{\mu} \frac{Y}{2} f_{I I I}\right) \\
& +\frac{4 \pi \alpha_{Y}}{M_{Z^{\prime}}^{2}}\left(\bar{f}_{I I} \gamma^{\mu} \frac{Y}{2} f_{I I}\right)\left(\bar{f}_{I I I} \gamma_{\mu} \frac{Y}{2} f_{I I I}\right)
\end{aligned}
$$


where $q$ is any quark, $f$ is a quark or lepton whose subscript indicates its generation, the $\lambda^{a}$ are the octet of Gell-Mann matrices, and Y is the standard model hypercharge generator円. The coefficients $\kappa_{1}$ and $\kappa_{3}$ are defined as

$$
\kappa_{1}=\alpha_{Y}\left(\frac{g_{1_{(1)}}}{g_{1_{(2)}}}\right)^{2} \quad \kappa_{3}=\alpha_{s}\left(\frac{g_{3_{(1)}}}{g_{3_{(2)}}}\right)^{2} .
$$

Note that $g_{i_{(1)}} / g_{i_{(2)}} \equiv \cot \left(\theta_{i}\right)$ where $\theta_{i}$ is the angle by which the original color $(\mathrm{i}=3)$ and hypercharge $(\mathrm{i}=1)$ gauge eigenstates were rotated to form the mass eigenstates.

The extended gauge interactions are ultimately responsible for the large mass of the top quark. The principle contributions to the dynamical mass come from the four-fermion contact interactions (3.1) and (3.2), which we can study using the gap equation in the Nambu-Jona-Lasinio (NJL) approximation [15]. The dynamical mass of fermion $f$ is the solution to:

$$
m_{f}=G_{1} \frac{m_{f} M_{Z^{\prime}}^{2}}{8 \pi^{2}}\left[1-\left(\frac{m_{f}}{M_{Z^{\prime}}}\right)^{2} \ln \left(\frac{M_{Z^{\prime}}^{2}}{m_{f}^{2}}\right)\right]+G_{3} \frac{3 m_{f} M_{C}^{2}}{8 \pi^{2}}\left[1-\left(\frac{m_{f}}{M_{C}}\right)^{2} \ln \left(\frac{M_{C}^{2}}{m_{f}^{2}}\right)\right]
$$

where the coefficients $G_{i}$ are

$$
\begin{aligned}
G_{3} & =0 \text { for leptons } \quad G_{3}=4 \pi \frac{\kappa_{3}}{M_{C}^{2}} \text { for quarks } \\
G_{1} & =\frac{2 \pi \alpha_{Y}^{2}}{M_{Z^{\prime}}^{2} \kappa_{1}} Y_{L}^{f} Y_{R}^{f} \text { for generations I and II } \\
G_{1} & =\frac{2 \pi \kappa_{1}}{M_{Z^{\prime}}^{2}} Y_{L}^{f} Y_{R}^{f} \text { for generation III }
\end{aligned}
$$

and $Y_{L}^{f}\left(Y_{R}^{f}\right)$ is the hypercharge of $f_{L}\left(f_{R}\right)$. In solving (3.4), we take the cut-off $\Lambda$ for the gap equation to be of order the coloron and $Z^{\prime}$ masses: $\Lambda \sim M_{C} \sim M_{Z^{\prime}}$; corrections due to unequal values for the coloron and $Z^{\prime}$ masses are small in the region of physical interest. Applying this to the top quark, one finds $\langle\bar{t} t\rangle \neq 0$ if

$$
\kappa_{3}+\frac{2}{27} \kappa_{1} \geq \frac{2 \pi}{3}
$$

More generally, however, we need to include contributions to the gap equation from gluon and hypercharge boson exchangef in effect, we are studying a gauged NJL model [16]. As discussed in [17], this modifies the criticality conditions for the $\kappa_{i}$.

Applying the gauged NJL gap equations to all the standard model fermions, we seek solutions with non-zero $m_{t}$ (i.e. formation of a top condensate $\langle\bar{t} t\rangle \neq 0$ ) and no mass for any other fermion (i.e. $\langle\bar{f} f\rangle=0$ for $f \neq t$ ). Such solutions exist

\footnotetext{
${ }^{1}$ We use the convention $Q=T_{3}+\frac{1}{2} Y$.

${ }^{2}$ Since the $S U(2)_{W}$ bosons couple only to left-handed fermions, they do not contribute here.
} 
provided that $\kappa_{1}$ and $\kappa_{3}$ satisfy a set of inequalities, of which the following three are the most stringent:

$$
\begin{aligned}
\kappa_{3}+\frac{2}{27} \kappa_{1} & \geq \frac{2 \pi}{3}-\frac{4}{3} \alpha_{s}-\frac{4}{9} \alpha_{Y} \\
\kappa_{3}+\frac{2}{27} \frac{\alpha_{Y}^{2}}{\kappa_{1}} & <\frac{2 \pi}{3}-\frac{4}{3} \alpha_{s}-\frac{4}{9} \alpha_{Y} \\
\kappa_{1} & <2 \pi-6 \alpha_{Y} .
\end{aligned}
$$

Inequality (3.6) leads to top quark condensation $(\langle\bar{t} t\rangle \neq 0)$. Note how including the effects of gluon and hypercharge boson exchange modifies the right-hand-side expression compared to the original NJL result (11). Inequality (3.7) implies $\langle\bar{c} c\rangle=0$ (i.e., no charm quark condensation). In our class of models, this is a stronger constraint than the inequality ensuring $\langle\bar{b} b\rangle=0$; in a Top-color I model [10], the latter would be the relevant constraint. Inequality (3.8) is related to the lack of $\tau$ condensation; it will be superseded by other constraints later in our discussion.

As inequalities (3.6) - (3.8) can be simultaneously satisfied, our models $d o$ admit the possibility that only the top quark condenses and receives an enhanced mass. The values of the couplings $\kappa_{1}$ and $\kappa_{3}$ for which this happens fall within the 'gap triangle 3 lying to the right of curve (1), to the left of curve (2) and below curve (3) in Figure 1 (by analogy with results for Top-color I models [10]). Solutions to the gauged-NJL gap equation [18] for $m_{t}=175 \mathrm{GeV}$ and particular values of the cut-off $\Lambda \sim M_{C} \sim M_{Z^{\prime}}$ lie on curves parallel to curve (1); a few examples for $\Lambda$ ranging from $0.7 \mathrm{TeV}$ to $5 \mathrm{TeV}$ are shown and labeled (A) through (D). Curves like these will be used in calculating phenomenological limits in the next section.

\section{Low-energy constraints}

We now consider how several types of physics constrain the allowed region of the $\kappa_{1}-\kappa_{3}$ plane. We first look at the $\rho$ parameter and $Z$ decays to tau leptons. Next, we discuss the implications of a strong $U(1)_{1}$ coupling. Finally, we comment on flavor-changing neutral currents (FCNC).

Current measurements of the $\rho$ parameter are already sensitive to the presence of the low energy contact interactions (3.1) and (3.2). The main contribution to $\Delta \rho_{*}$ from the coloron sector of our model is [19] single coloron exchange across the top and bottom quark loops of $W$ and $Z$ vacuum polarization diagrams. Applying the results of [19] to our models, we have

$$
\Delta \rho_{*}^{(C)} \approx \frac{16 \pi^{2} \alpha_{Y}}{3 \sin ^{2} \theta_{W}}\left(\frac{f_{t}^{2}}{M_{C} M_{Z}}\right)^{2} \kappa_{3}
$$

\footnotetext{
${ }^{3}$ Due to the non-linearity of (3.7), it is only approximately a triangle.
} 


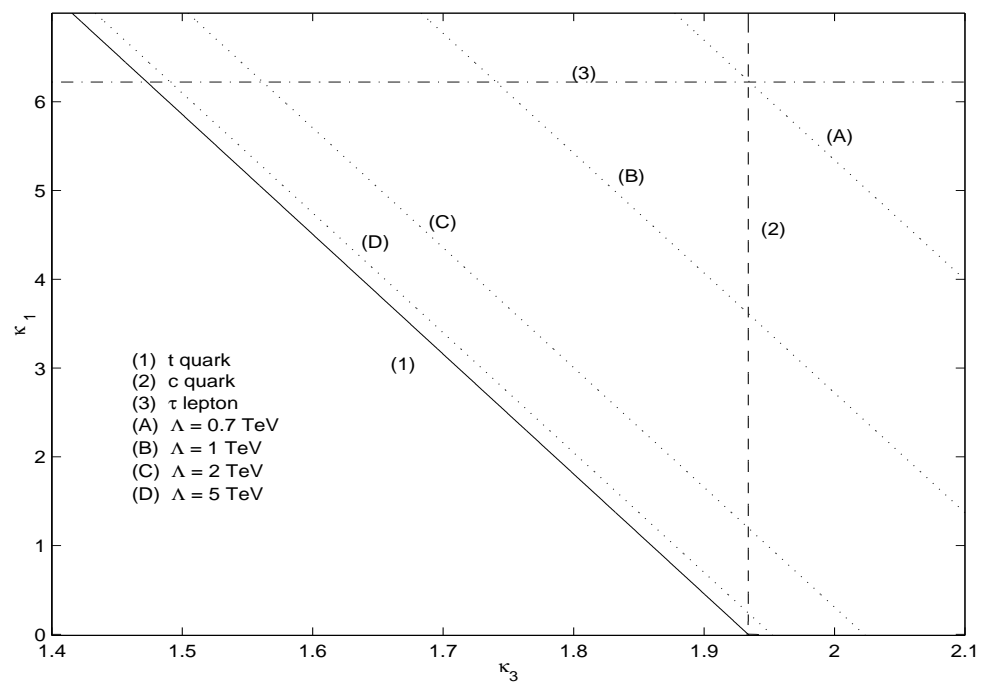

Figure 1: The gap triangle, bounded by curves (1), (2), and (3) is the region within which only the top quark condenses. Above curve $(1)\langle\bar{t} t\rangle \neq 0$; to the left of curve $(2)\langle\bar{c} c\rangle=0$, and below curve $(3)\langle\bar{\tau} \tau\rangle=0$. Lines $(\mathrm{A}, \mathrm{B}, \mathrm{C}, \mathrm{D})$ represent solutions to the gap equation [18] for $m_{t}=175$ assuming $\Lambda \sim M_{Z^{\prime}} \sim M_{C}$ has values of $(0.7,1.0,2.0,5.0) \mathrm{TeV}$.

where $\theta_{W}$ is the weak mixing angle and $f_{t}$ is the analog of $f_{\pi}$ for the top-condensate, i.e. (in the NJL approximation) [15, 20]

$$
f_{t}^{2}=\frac{3}{8 \pi^{2}} m_{t}^{2} \ln \left(\frac{\Lambda^{2}}{m_{t}^{2}}\right)
$$

In the $Z^{\prime}$ sector, the main contribution to $\Delta \rho_{*}$ arises from $Z-Z^{\prime}$ mixing. Adapting the results of [21] to our models, we have

$$
\Delta \rho_{*}^{\left(Z^{\prime}\right)} \approx \frac{\alpha_{Y} \sin ^{2} \theta_{W}}{\kappa_{1}} \frac{M_{Z}^{2}}{M_{Z^{\prime}}^{2}}\left[1-\frac{f_{t}^{2}}{v^{2}}\left(\frac{\kappa_{1}}{\alpha_{Y}}+1\right)\right]^{2} .
$$

Requiring $\Delta \rho_{*}=\Delta \rho_{*}^{(C)}+\Delta \rho_{*}^{\left(Z^{\prime}\right)}<0.4 \%$ 19] excludes the region to the right of curve (4) in Figure 2. This curve connects the points $\Delta \rho_{*}=0.4 \%$ on the lines of constant $\Lambda \sim M_{C} \sim M_{Z^{\prime}}$ mentioned earlier. Note how the $\Delta \rho_{*}$ constraint narrows the allowed region of the $\kappa_{1}-\kappa_{3}$ plane.

Another constraint comes from the partial decay width of the $\mathrm{Z}$ boson to tau leptons:

$$
\Gamma\left(Z \rightarrow \tau^{+} \tau^{-}\right)=\frac{G_{F} M_{Z}^{3}}{3 \sqrt{2} \pi}\left[g_{\tau_{L}}^{2}+g_{\tau_{R}}^{2}\right]
$$

where $G_{F}$ is the Fermi constant [5] and $g_{\tau_{L}}\left(g_{\tau_{R}}\right)$ is the coupling of $\tau_{L}\left(\tau_{R}\right)$ to the $Z$ boson. Due to $Z-Z^{\prime}$ mixing, [21], the couplings $g_{\tau_{L}}$ and $g_{\tau_{R}}$ in our model are 


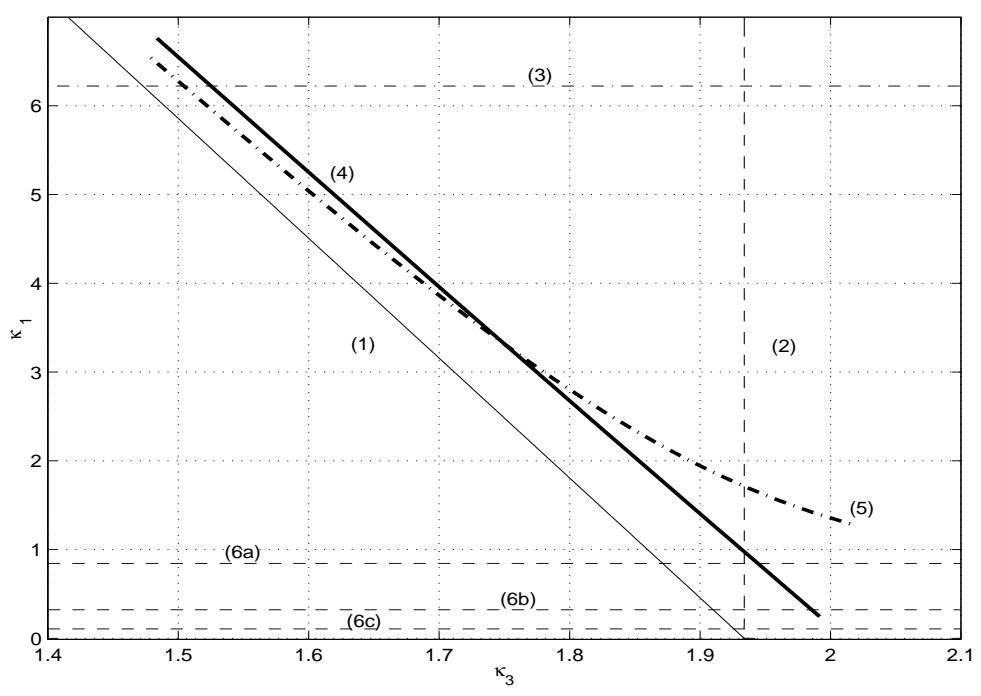

Figure 2: Low-energy constraints. Curves (1), (2), (3) outline the 'gap triangle' of Figure 1 where only $\langle\bar{t} t\rangle \neq 0$. The region above curve (4) is excluded by data on $\Delta \rho_{*}$; the region above curve (5) is excluded by data on $Z \rightarrow \tau^{+} \tau^{-}$. Lines (6a-6c) are possible upper bounds on $\kappa_{1}$ from triviality as in Figure 3.

altered from those in the standard model (i.e. $g_{\tau} \rightarrow g_{\tau}(S M)+\delta g_{\tau}$ ) by

$$
\delta g_{\tau_{L}}=\frac{1}{2} \delta g_{\tau_{R}}=\sin ^{2} \theta_{W} \frac{M_{Z}^{2}}{M_{Z^{\prime}}^{2}}\left[1-\frac{f_{t}^{2}}{v^{2}}\left(\frac{\kappa_{1}}{\alpha_{Y}}+1\right)\right]
$$

yielding a non-standard prediction for $\Gamma\left(Z \rightarrow \tau^{+} \tau^{-}\right)$. Including QED corrections to eq. (4.4) and requiring our predicted value to be consistent with the experimental [5] value $\Gamma^{\operatorname{expt}}\left(Z \rightarrow \tau^{+} \tau^{-}\right)=83.67 \pm 0.44 \mathrm{MeV}$ at $95 \%$ c.l. excludes the region to the right of curvet (5) in Figure 2.

The asymptotic UV behavior of the strongly-coupled $U(1)_{1}$ yields another important, albeit elastic, constraint on $\kappa_{1}$. Combining expressions (2.4) and (3.3) shows that

$$
\frac{g_{1}^{2}}{4 \pi}=\alpha_{Y}+\kappa_{1}
$$

Applying the renormalization group equation to $U(1)_{1}$

$$
\left.\frac{g_{1(1)}^{2}}{4 \pi}\right|_{\Lambda_{H}}=\frac{\left.\frac{g_{1_{(1)}^{2}}^{2}}{4 \pi}\right|_{\Lambda}}{1-\left.\left(\frac{g_{(1)}^{2}}{4 \pi}\right)\right|_{\Lambda} \frac{C}{3 \pi} \ln \left(\frac{\Lambda_{H}^{2}}{A \Lambda^{2}}\right)}
$$

\footnotetext{
${ }^{4}$ This curve was constructed by the same procedure as curve (4).

${ }^{5}$ We thank R.S. Chivukula for emphasizing the relevance of this constraint. Similar considerations apply in any model in which a $U(1)$ gauge interaction is used to align the vacuum 22].
} 


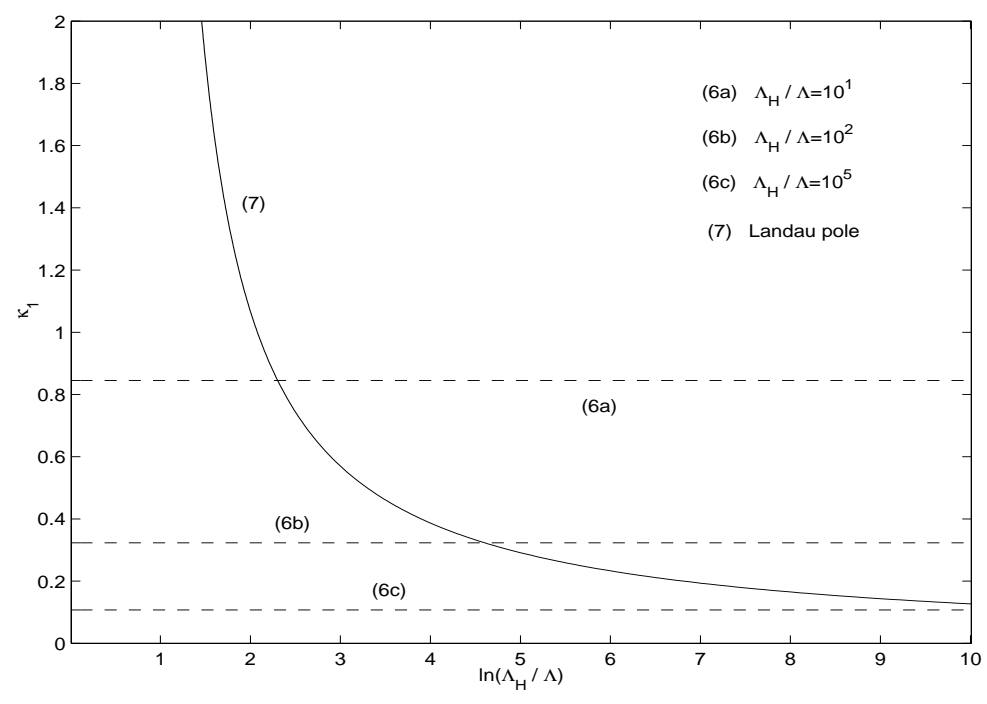

Figure 3: The position of the Landau pole $\Lambda_{H}$ for $U(1)_{1}$ is shown by curve (7). Lines (6a-6c) show the upper bound on $\kappa_{1}$ that holds if the Landau pole lies one, two or five orders of magnitude above $\Lambda$; these also appear in Figure 2.

(with $A=\exp \left(\frac{5}{3}\right)$ ) and considering just the contribution from the standard model particles (i.e., taking $C=\frac{15}{4}$ ) allows us to estimate the position of the Landau pole for a given low-energy value of $\kappa_{1}$. Our results are summarized in Figure 3 . If the Landau pole is to lie at least an order of magnitude above the symmetry-breaking scale, $\Lambda$, then $\kappa_{1}$ must be of order 1 or smaller. This defines curve (6a) in Figures 2 and 3. Similarly, requiring the Landau pole to lie two or five orders of magnitude above $\Lambda$ produces curves (6b) and (6c) in Figures 2 and 3.

Finally, we turn to flavor-changing neutral currents. Because the color sector is flavor-universal, the low-energy effective interactions (3.1) cause no flavor-changing neutral currents. In other words, the low-energy effective theory now includes not just top-pions [8], but a complete set of "q-pions" strongly coupled to all flavors of quarks. To first approximation, the q-pion masses and couplings are flavorsymmetric and they make no contribution to hadronic FCNC processes like neutral meson mixing or $b \rightarrow s \gamma$. This is in contrast to the potentially large (but avoidable) hadronic FCNC exhibited by Top-color I models [23, 10]. The flavor symmetry among the q-pions will be modified at sub-leading level by non-universal U(1) effects; this can re-introduce hadronic FCNC at a smaller, less dangerous rate.

Because the hypercharge interactions (3.2) distinguish among generations, they also cause semi-leptonic flavor-changing decays of $B$ and $K$ mesons, which are the same as those in Top-color I models [10]. As discussed in ref. [10], current data on $B_{s} \rightarrow l^{+} l^{-}, B \rightarrow X_{s} l^{+} l^{-}, B \rightarrow X_{s} \nu \bar{\nu}$, and also $ๆ \Upsilon(4 S) \rightarrow l^{+} l^{-}$set no limits,

\footnotetext{
${ }^{6}$ While this process involves no FCNC, it would be similarly affected by the $Z^{\prime}$ boson.
} 
but future experiments may be sensitive to the presence of the additional interactions. For the process $K^{+} \rightarrow \pi^{+} \nu_{\tau} \bar{\nu}_{\tau}$, ref. [10] found that the ratio of amplitudes was roughly $\left|A_{\text {new }} / A_{S M}\right| \sim 1.5 \kappa_{1} / M_{Z^{\prime}}^{2} \mathrm{TeV}^{2}$, so squaring this and dividing by the number of neutrino species gives an estimate of the relative branching ratios: $B_{\text {new }}\left(K \rightarrow \pi \nu_{\tau} \bar{\nu}_{\tau}\right) / B_{S M}(K \rightarrow \pi \nu \bar{\nu}) \sim 0.8\left(\kappa_{1} / M_{Z^{\prime}}^{2}\right)^{2} \mathrm{TeV}^{4}$. Subsequently, evidence has been published for a $K^{+} \rightarrow \pi^{+} \nu \bar{\nu}$ event that is consistent with branching ratio $4.2_{-3.5}^{+9.7} \times 10^{-10}$ [24], as compared with a standard model branching ratio of order $10^{-10}$. This process is therefore still able to accommodate a $Z^{\prime}$ in the allowed parameter space of our models (i.e., $\kappa_{3} \approx 2$ and $\kappa_{1} \lesssim 1$ ); future data from the E787 Collaboration may provide further constraints.

\section{$5 \quad$ Direct Searches for the Colorons and $Z^{\prime}$}

The colorons in this class of models are identical to those introduced in the flavoruniversal coloron model of ref. [12]. As discussed in 25], searches in dijet final states should be the most powerful way of locating heavy colorons. Searches in $b \bar{b}$ and $t \bar{t}$ offer no particular advantage in searching for the flavor-universal colorons in our class of models. This is in contrast to the case of the topgluons of topcolor [0] and topcolor-assisted technicolor [8].

As discussed earlier, constraints on the low-energy effective theory for our class of models limit the value of coupling $\kappa_{3}$ to lie quite close to the critical value $\approx 2$. This means that the coloron cannot be very light: if we estimate the minimum coloron mass for $\kappa_{3}=2$ by requiring the coloron contribution (4.1) to $\Delta \rho^{*}$ to be less than $0.4 \%$, we find $M_{c} \gtrsim 1.6 \mathrm{TeV}$; including the $Z^{\prime}$ contributions to $\Delta \rho^{*}$ would only strengthen the bound. A coloron of this large a mass lies above the reach of published searches for new particles decaying to dijets [26]. Moreover, the large value of $\kappa_{3}$ implies that the coloron's width

$$
\Gamma_{C} \approx M_{C} \kappa_{3}\left[\frac{5}{6}+\frac{1}{6}\left(1-\frac{m_{t}^{2}}{M_{C}^{2}}\right) \sqrt{1-\frac{4 m_{t}^{2}}{M_{C}^{2}}}\right]
$$

is approximately twice its mass. Future searches for narrow resonances will not be appropriate for finding these colorons. A more promising approach would employ the strategies of compositeness searches, which focus on high- $E_{T}$ enhancement of single-jet inclusive and dijet spectra [27] or alteration of the dijet angular distributions [28]. At energies well below $M_{C}$, the effects of coloron exchange on hadronic scattering are approximated by those of the color-octet quark contact interaction (3.1). If experiment set a limit $\Lambda_{\text {octet }}>X \mathrm{TeV}$ on a color-octet contact interaction

$$
-\frac{g_{o}^{2}}{2 ! \Lambda_{\text {octet }}^{2}}\left(\bar{q} \gamma^{\mu} \frac{\lambda^{a}}{2} q\right)\left(\bar{q} \gamma_{\mu} \frac{\lambda_{a}}{2} q\right)
$$

with the usual convention $g_{o}^{2} / 4 \pi \equiv 1$, this would imply a limit $M_{C}>\sqrt{2} X \mathrm{TeV}$ for our class of models in which $\kappa_{3} \approx 2$. 
Existing limits on the mass of the $Z^{\prime}$ boson are not very stringent. For example, Tevatron bounds [29] on new contributions to the dilepton $(e e$ or $\mu \mu)$ mass spectrum from interactions like (3.2) set no useful limit on our class of models because the $Z^{\prime}$ coupling to first generation fermions is so small. The strongest limits are derived in ref. [21] by considering the contributions to electroweak observables of a $Z^{\prime}$ like the one in our class of models (called an "optimal" $Z^{\prime}$ in [21]). These calculations set a $95 \%$ c.l. lower bound of $290 \mathrm{GeV}$ on the $Z^{\prime}$ for $\kappa_{1} \approx 0.13$. For other values of $\kappa_{1}$, the $Z^{\prime}$ must be heavier; a $Z^{\prime}$ mass less than a TeV is allowed for $.014 \lesssim \kappa_{1} \lesssim .23$. Including the effects of the colorons and q-pions on electroweak observables would presumably strengthen the lower bounds on $M_{Z^{\prime}}$, as coloron exchange tends to increase $\Delta \rho^{*}$ (c.f. 4.1) and the q-pions will contribute to hadronic $Z$ decays]

Future experiments measuring production of third-generation fermions $\left(\tau^{+} \tau^{-}\right.$, $\bar{b} b, \bar{t} t)$ have the greatest potential to find signs of the $Z^{\prime}$ boson. Consider, for example, looking for an excess in $e^{+} e^{-} \rightarrow \tau^{+} \tau^{-}$in $50 \mathrm{fb}^{-1}$ of NLC data taken at $\sqrt{s}=500 \mathrm{GeV}$. Because the $Z^{\prime}$ boson's decay width

$$
\Gamma_{Z^{\prime}}=M_{Z^{\prime}} \frac{\kappa_{1}}{3}\left[\frac{20}{3}\left(\frac{\alpha_{Y}}{\kappa_{1}}\right)^{2}+\frac{23}{12}+\frac{17}{12}\left(1-\frac{m_{t}^{2}}{M_{Z^{\prime}}^{2}}\right) \sqrt{1-\frac{4 m_{t}^{2}}{M_{Z^{\prime}}^{2}}}\right]
$$

is a large fraction of its mass (e.g., $\Gamma_{Z^{\prime}} \approx .5 M_{Z^{\prime}}$ for $\kappa_{1}=.5$ ), we use the $s$-dependent width in the cross-section; this renders our results insensitive to the exact value of $\kappa_{1}$. Assuming a $50 \%$ efficiency for identifying tau pairs and requiring a excess over the standard model prediction for $e^{+} e^{-} \rightarrow[\gamma, Z] \rightarrow \tau^{+} \tau^{-}$of $\left(N^{\tau \tau}-N_{S M}^{\tau \tau}\right) \geq 5 \sqrt{N_{S M}^{\tau \tau}}$, the effects of a $2.7 \mathrm{TeV} Z^{\prime}$ boson with $\kappa_{1} \leq 1$ could be visible. At a $1.5 \mathrm{TeV}$ NLC with $200 \mathrm{fb}^{-1}$ of data, the reach in $M_{Z^{\prime}}$ extends to $6.6 \mathrm{TeV}$.

\section{Conclusions}

We have examined the low-energy effective theory and phenomenology of a class of technicolor models with flavor-universal extended color interactions and a generationdistinguishing extended hypercharge sector. Such models are found to be capable of dynamically producing a top quark condensate that preferentially enhances the mass of the top quark. Moreover, flavor-changing neutral currents are less dangerous here than in models where the color sector couples differently to the third generation. Constraints from $Z$-pole physics and $U(1)$ triviality single out the region of coupling-constant parameter space where $\kappa_{3} \approx 2$ and $\kappa_{1} \lesssim 1$ for further study. Electroweak physics presently constrains the $Z^{\prime}$ boson in these models to weigh at least $290 \mathrm{GeV}$, while the octet of flavor-universal colorons must have a mass of at least $1.6 \mathrm{TeV}$. Future studies of jet physics at hadron colliders have the potential to

\footnotetext{
${ }^{7}$ Indeed, the presence of a full set of q-pions offers the possibility of new effects controlled by the scale $M_{q-\text { pion }}$ that may offset the large negative contributions to $R_{b}$ from top-pions and bottompions (and similar effects on $R_{c}$ ) found in [30] for topcolor models. This will be addressed in future work.
} 
uncover evidence of the colorons, while data on pair-production of third-generation fermions at $e^{+} e^{-}$machines can help discover the $Z^{\prime}$.

\section{Acknowledgments}

We thank R.S. Chivukula, N. Evans, C.T. Hill, K.D. Lane, and T. Rizzo for useful discussions and comments on the manuscript. E.H.S. is grateful for the hospitality of the Aspen Center for Physics during the inception of this work and that of the Theoretical Physics Group at Fermilab during its completion. E.H.S. acknowledges the support of the Faculty Early Career Development (CAREER) program and the DOE Outstanding Junior Investigator program. This work was supported in part by the National Science Foundation under grant PHY-9501249, and by the Department of Energy under grant DE-FG02-91ER40676.

\section{References}

[1] S. Weinberg, Phys. Rev. D13 (1976) 974; 19 (1979) 1277; L. Susskind, Phys. Rev. D20 (1979) 2619.

[2] S. Dimopoulos and L. Susskind, Nucl. Phys. B155 (1979) 237; E. Eichten and K. Lane, Phys. Lett. B90 (1980) 125.

[3] T. Appelquist et al., Phys. Rev. Lett. 53 (1984) 1523.

[4] R. S. Chivukula, S. B. Selipsky and E. H. Simmons, Phys. Rev. Lett. 69 (1992) 575, hep-ph/9204214.

[5] Particle Data Group, R.M. Barnett et al., Physical Review D54, 1 (1996) and 1997 off-year partial update for the 1998 edition available on the PDG WWW pages (URL: http://pdg.lbl.gov/).

[6] V.A. Miransky, M. Tanabashi, and K. Yamawaki, Phys. Lett. B221 (1989) 177 and Mod. Phys. Lett. A4 (1989) 1043; Y. Nambu, Chicago preprint EFI 89-08 (1989); W.J. Marciano, Phys. Rev. Lett. 62 (1989) 2793 and Phys. Rev. D41 (1990) 219; W.A. Bardeen, C.T. Hill and M. Lindner, Phys. Rev. D41 (1990) 1647.

[7] C.T. Hill, Phys. Lett. B266 (1991) 419

[8] C.T. Hill, Phys. Lett. B345 (1995) 483, hep-ph/9411426

[9] K. Lane and E. Eichten, Phys. Lett. B352 (1995) 382, hep-ph/9503433; K.D. Lane, Phys. Rev. D54 (1996) 2204, hep-ph/9602221

[10] G. Buchalla et al., Phys. Rev. D53 (1996) 5185, hep-ph/9510376. 
[11] R.S. Chivukula, E.H. Simmons, J. Terning, Phys. Lett. B331 (1994) 383, hep-ph/9404209; R.S. Chivukula, E.H. Simmons, J. Terning, Phys. Rev. D53 (1996) 5258, hep-ph/9506427; E.H. Simmons, Phys. Rev. D55 (1997) 5494, hep-ph/9612402.

[12] R.S. Chivukula, A.G. Cohen and E.H. Simmons, Phys. Lett. B380 (1996) 92, hep-ph/9603311

[13] K.D. Lane, "A new model of topcolor assisted technicolor," BUHEP-98-9, hep$\mathrm{ph} / 9805254$.

[14] C.T. Hill and S.J. Parke, Phys. Rev. D49 (1994) 4454, hep-ph/9312324.

[15] Y. Nambu and G. Jona-Lasinio, Phys. Rev. 122 (1961) 345; Y. Nambu and G. Jona-Lasinio, Phys. Rev. 124 (1961) 246.

[16] W.A. Bardeen, C.N. Leung and S.T. Love, Phys. Rev. Lett. 56 (1986) 1335; C.N. Leung, S.T. Love and W.A. Bardeen, Nucl. Phys. B273 (1986) 649.

[17] T. Appelquist et al., in Proceedings of Johns Hopkins Workshop on Current Problems in Particle Theory, eds. G. Domokos and S. Kovesi-Domokos (World Scientific Publishing Col, Singapore, 1988); K.-I. Kondo, H. Mino and K. Yamawaki, Phys. Rev. D39 (1989) 2430; for a review, see V. Miransky, Dynamical Symmetry Breaking in Quantum Field Theories, (World Scientific Publishing Co., Singapore, 1993).

[18] T. Nonoyama, T. Suzuki, and K. Yamawaki, Prog. Theor. Phys. 81 (1989) 1238.

[19] R. S. Chivukula, B. A. Dobrescu, J. Terning, Phys. Lett. B353 (1995) 289, hep-ph/9503203.

[20] H. Pagels and S. Stokar, Phys. Rev. D20 (1979) 2947.

[21] R. S. Chivukula, J. Terning, Phys. Lett. B385 (1996) 209, hep-ph/9606233.

[22] R.S. Chivukula and H. Georgi, "Effective Field Theory of Vacuum Tilting," BUHEP-98-15, HUTP-98/A042. hep-ph/9608289.

[23] D. Kominis, Phys. Lett. B358 (1995) 312, hep-ph/9506305.

[24] E787 Collaboration, S. Adler et al., Phys. Rev. Lett. 79 (1997) 2204, hepex/9708031.

[25] E.H. Simmons, Phys. Rev. D55 (1997) 1678, hep-ph/9608269.

[26] CDF Collaboration, F. Abe et al., Phys. Rev. Lett. 55 (1997) R5263, hepex/9702004; UA1 Collaboration, C. Albajar et al., Phys. Lett. B209 (1988) 127; UA2 Collaboration, Phys. Lett. B400 (1993) 3. 
[27] CDF Collaboration, F. Abe et al., Phys. Rev. Lett. 77 (1996) 438, hepex/9601008.

[28] D0 Collaboration, B. Abbott et al., Phys. Rev. Lett. 80 (1998) 666, hepex/9707016; CDF Collaboration, F. Abe et al., Phys. Rev. Lett. 77 (1996) 5336 and Erratum 78 (1997) 4307, hep-ex/9609011.

[29] CDF Collaboration, F. Abe et al., Phys. Rev. Lett. 79 (1997) 2198.

[30] G. Burdman, and D. Kominis Phys. Lett. B403 (1997) 101, hep-ph/9702265. 